\title{
BURNING PLASMA PROJECTIONS USING DRIFT WAVE TRANSPORT MODELS AND SCALINGS FOR THE H-MODE PEDESTAL
}

\author{
by \\ J.E. KINSEY, T. ONJUN, G. BATEMAN, A. KRITZ, \\ A. PANKIN, G.M. STAEBLER, and R.E. WALTZ
}




\section{DISCLAIMER}

This report was prepared as an account of work sponsored by an agency of the United States Government. Neither the United States Government nor any agency thereof, nor any of their employees, makes any warranty, express or implied, or assumes any legal liability or responsibility for the accuracy, completeness, or usefulness of any information, apparatus, product, or process disclosed, or represents that its use would not infringe privately owned rights. Reference herein to any specific commercial product, process, or service by trade name, trademark, manufacturer, or otherwise, does not necessarily constitute or imply its endorsement, recommendation, or favoring by the United States Government or any agency thereof. The views and opinions of authors expressed herein do not necessarily state or reflect those of the United States Government or any agency thereof. 


\title{
BURNING PLASMA PROJECTIONS USING DRIFT WAVE TRANSPORT MODELS AND SCALINGS FOR THE H-MODE PEDESTAL
}

\author{
by \\ J.E. KINSEY, ${ }^{*}$ T. ONJUN, ${ }^{*}$ G. BATEMAN, ${ }^{*}$ A. KRITZ, ${ }^{*}$ \\ A. PANKIN, ${ }^{*}$ G.M. STAEBLER, and R.E. WALTZ
}

This is a preprint of a paper presented at the Nineteenth IAEA Fusion Energy Conference, Lyon, France, October 14-19, 2002, and to be published on CD-ROM.

${ }_{1}$ LeHigh University, Bethlehem, Pennsylvania

Work supported by

U.S. Department of Energy under

Grant Nos. DE-FG03-95ER54309

and DE-FG02-92ER54141 


\section{ABSTRACT}

The GLF23 [1] and Multi-Mode (MM95) [2] transport models are used along with a model for the $\mathrm{H}$-mode pedestal to predict the fusion performance for the ITER, FIRE, and IGNITOR tokamak designs. The drift-wave predictive transport models reproduce the core profiles in a wide variety of tokamak discharges, yet they differ significantly in their response to temperature gradient (stiffness). Recent gyro-kinetic simulations of ITG/TEM [3] and ETG modes [4] motivate the renormalization of the GLF23 model. The normalizing coefficients for the ITG/TEM modes are reduced by a factor of 3.7 while the ETG mode coefficient is increased by a factor of 4.8 in comparison with the original model. A pedestal temperature model is developed for type I ELMy H-mode plasmas based on ballooning mode stability and a theory-motivated scaling for the pedestal width. In this pedestal model, the pedestal density is proportional to the line-averaged density and the pedestal temperature is inversely related to the pedestal density.

In an effort to provide predictive boundary conditions for integrated modeling simulations, a model has been developed to predict the temperature and density at the top of the pedestal at the edge of type I ELMy H-mode tokamak plasmas. In the model for pedestal temperature, it is assumed that the edge pressure gradient is limited by high-n ideal MHD ballooning modes and the pedestal width scales like the major radius times the square root of poloidal beta, $\Delta \propto$ $\mathrm{R} \sqrt{ } \beta_{\mathrm{p}}$ [5]. The magnetic shear that is used in the ballooning mode limit is computed one pedestal width from the separatrix and is reduced by the effect of the bootstrap current. The pedestal temperature model is calibrated using 533 data points from the International Pedestal Database (v.3.1) based on experimental data from JT60-U, ASDEX-U, JET, and DIII-D. This model, shown in Fig. 1(a), has an RMS error of 33.5\%. The same data yields an empirical model for the pedestal density proportional to the line averaged density $\bar{n}_{e}\left(n_{p e d}=0.71 \bar{n}_{e}\right)$, which has an RMS error of $12.1 \%$, as shown in Fig. 1(b).

The GLF23 transport model [2] uses drift-wave linear eigenmodes to compute the quasilinear energy, toroidal momentum, and particle fluxes due to ion/electron temperature gradient (ITG/ETG) and trapped electron modes (TEM). The model differs from other drift-wave based transport models in that it includes kinetic effects through use of the gyro-Landau fluid equations. The transport is computed using a spectrum of eigenmodes with 10 wavenumbers for the ion temperature gradient (ITG) and trapped electron modes (TEM) and 10 wavenumbers for the short wavelength electron temperature gradient (ETG) modes. The fluxes were normalized to give the same ion thermal energy flux as non-linear gyro-Landau fluid simulations of ITG/TEM 

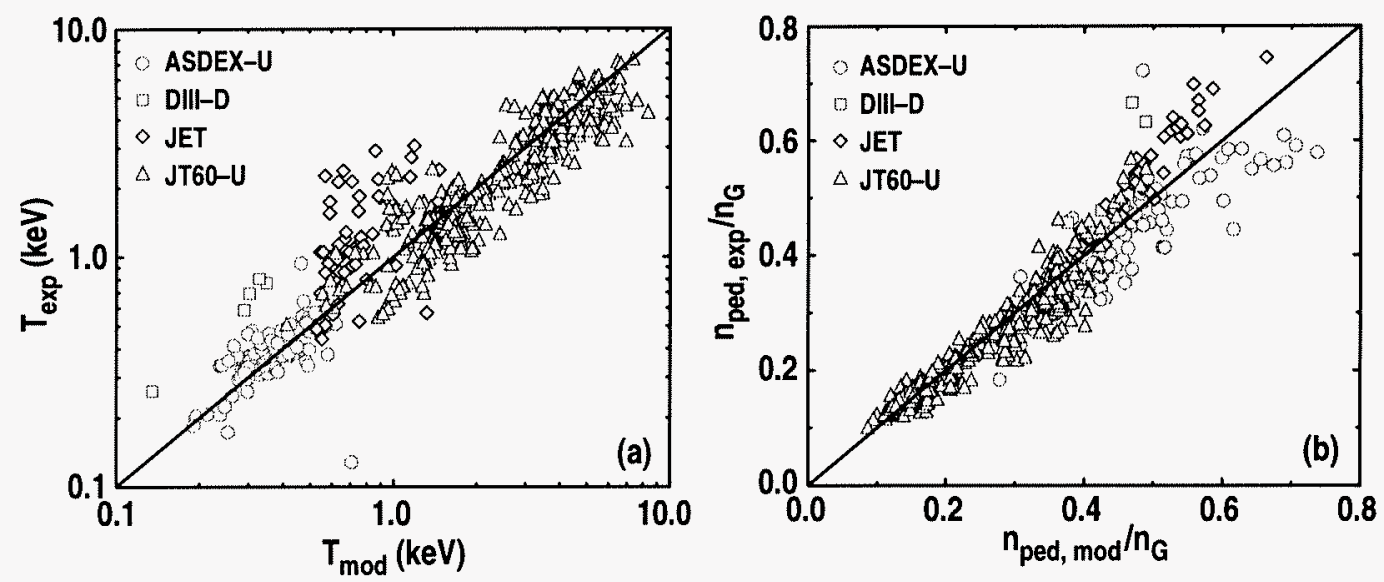

Fig. 1. (a) Pedestal temperatures from database compared with model predictions. (b) Pedestal density from database compared with model predictions.

modes. Since publication of the 1996 model, it has been found that fully kinetic non-linear simulations [3] predict a factor of 4 lower level of ITG transport than gyro-Landau fluid simulations for parameters used to normalize GLF23. Also, recent non-linear simulations of ETG modes show that the electron energy flux due to ETG modes can be much larger than estimated from simply rescaling ITG results using the electron to ion mass ratio [4]. However, it is not yet possible to perform a fully kinetic non-linear simulation with all of the physics included (e.g. both electrons and ions). It was therefore decided to determine the best fit value of the ETG mode coefficient in the model using experimental data. Since the proposed tokamak burning plasma experiments are based on the $\mathrm{H}$-mode operational regime, a database of $50 \mathrm{H}$-mode discharges from JET, DIII-D and C-Mod was used to determine the best fit value of the ETG mode coefficient. The ETG coefficient was varied and the best fit value determined to yield a zero offset in the predicted pedestal corrected stored energy (i.e. subtracting the stored energy outside the H-mode pedestal boundary condition) for the dataset. The normalizing coefficient for the ITG/TEM modes is reduced by a factor of 3.7 to be consistent with GYRO non-linear gyrokinetic simulations while the ETG mode coefficient is increased by factor of 4.8 compared with the original model. The quality of the fit is shown in Fig. 2(a). The renormalized GLF23 model has a standard deviation of only $\sigma=8.7 \%$ over the dataset which is a small improvement over the original model $(\sigma=10.0 \%)$.

The renormalized GLF23 model is still relatively stiff and the transport due to ITG modes tends to keep the ion temperature profile close to the critical gradient making the core temperature $\mathrm{T}_{0}$ track nearly linearly with the $\mathrm{H}$-mode pedestal temperature $\mathrm{T}_{\text {ped }}$. With the assumption that the transport is perfectly stiff, i.e. $\mathrm{T}_{0} \propto \mathrm{T}_{\text {ped }}$, the predicted fusion power can be

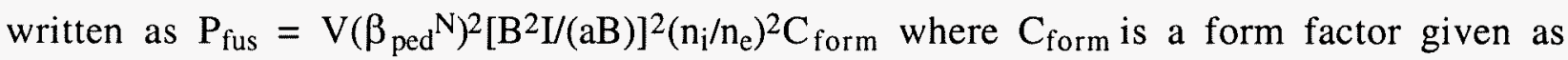


$\mathrm{K}\left(\overline{\mathrm{n}}_{\mathrm{e}} / \mathrm{n}_{\mathrm{ped}}\right)^{1.5} \mathrm{C}_{\mathrm{RLT} 1} \mathrm{C}_{\mathrm{RLT} 2}$ with $\mathrm{C}_{\mathrm{RLT} 1}=\exp \left[2\left(2.15+\left(1-\mathrm{n}_{\mathrm{i}} / \mathrm{n}_{\mathrm{e}}\right)+0.75\left(1+0.5 v^{-0.25}\right)\right) /(\mathrm{R} / \mathrm{a})\right]$ and $\mathrm{C}_{\mathrm{RLT} 2}=\exp \left[2\left(.00275 \mathrm{P}_{\text {net }}(\mathrm{R} / \mathrm{a})^{1.5} / \mathrm{T}_{\text {ped }}{ }^{1.5}\right)^{2}\right]$ as critical gradient correction factors. Here, $\mathrm{K}=6.7 \times 10^{-5}, \mathrm{P}_{\text {net }}=\mathrm{P}_{\mathrm{a}}+\mathrm{P}_{\mathrm{aux}}, \beta_{\text {ped }} \mathrm{N}^{\mathrm{N}}=\beta_{\mathrm{ped}} /(\mathrm{I} /(\mathrm{aB}))$, and $\mathrm{v}=<\mathrm{n}_{\mathrm{e}}>\mathrm{R} / \mathrm{T}_{\text {ped }}{ }^{2}$. The fusion power from the renormalized model (while not perfectly stiff) follows a universal stiff model curve that is machine independent and varies as $\left(\mathrm{T}_{\mathrm{ped}}\right)^{1.8}$ [see Fig. 2(b)].
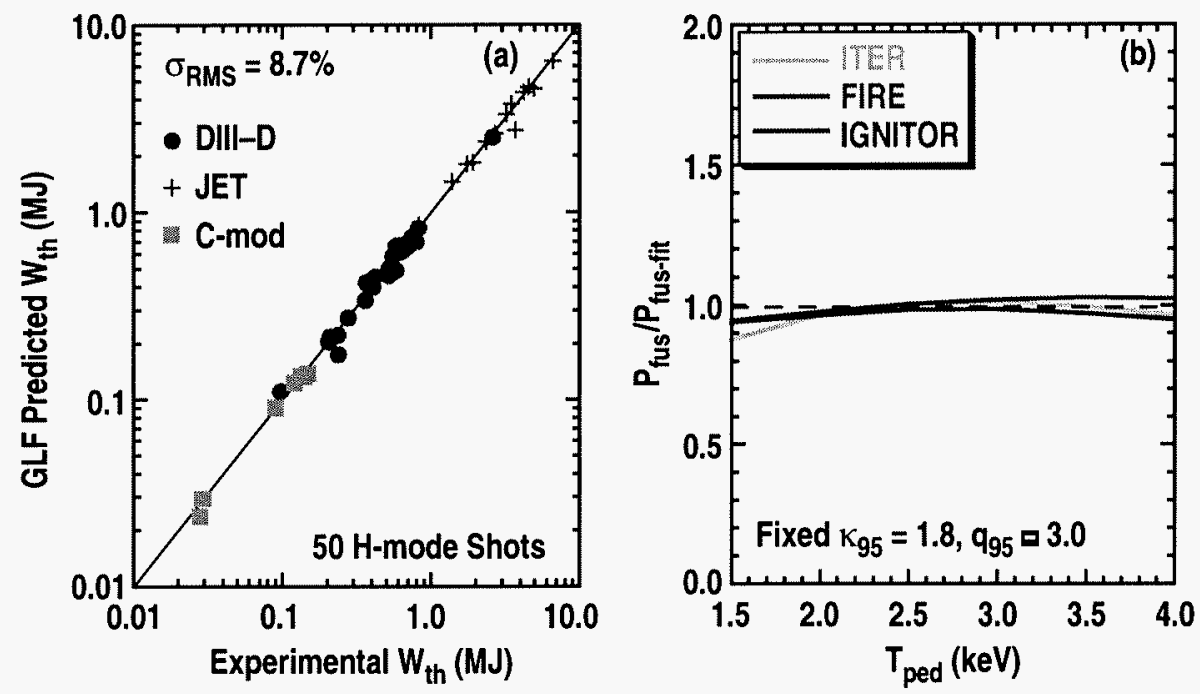

Fig. 2. (a) GLF23 predicted (renormalized) versus experimental core stored energy with boundary conditions enforced at $\rho=0.8$. (b) Normalized fusion power versus pedestal temperature at $\bar{n}_{e} / \mathrm{n}_{\mathrm{G}}=0.85,0.70$, and 0.50 for ITER, FIRE, and IGNITOR, respectively.

Predictive transport simulations are usually performed taking experimental data to determine the boundary conditions for the density, temperature, and toroidal rotation profiles. This limits the overall predictive capability of transport models. This issue is particularly important when considering fusion reactor performance involving $\mathrm{H}$-mode plasmas since the predicted fusion power is sensitive to the temperature and density values assumed at the top of the pedestal. The first pedestal model in Ref. [5], which is similar to the one described in this paper, has been used to provide the boundary conditions in simulations of $\mathrm{H}$-mode discharges using the MM95 model in the BALDUR code. It is found that the overall agreement between the modeled profiles and experimental data using the pedestal model previously described is approximately $10 \%$, which is nearly the same as the results obtained when the boundary conditions are prescribed using experimental data. A similar exercise using the renormed GLF23 model has been carried out using the pedestal scalings described in Ref. [6]. The results of using the power dependent thermal conduction pedestal scaling (Eq. (2) in Ref. [2]) to provide $T_{\text {ped }}$ in the simulations are shown in Fig. 3. For 47 of the $50 \mathrm{H}$-mode shots shown in Fig. 2(a), the power dependent scaling has an RMS error for $\mathrm{T}_{\text {ped }}$ of $33 \%$. Using $\mathrm{T}_{\text {ped }}$ from this pedestal scaling to set the boundary 

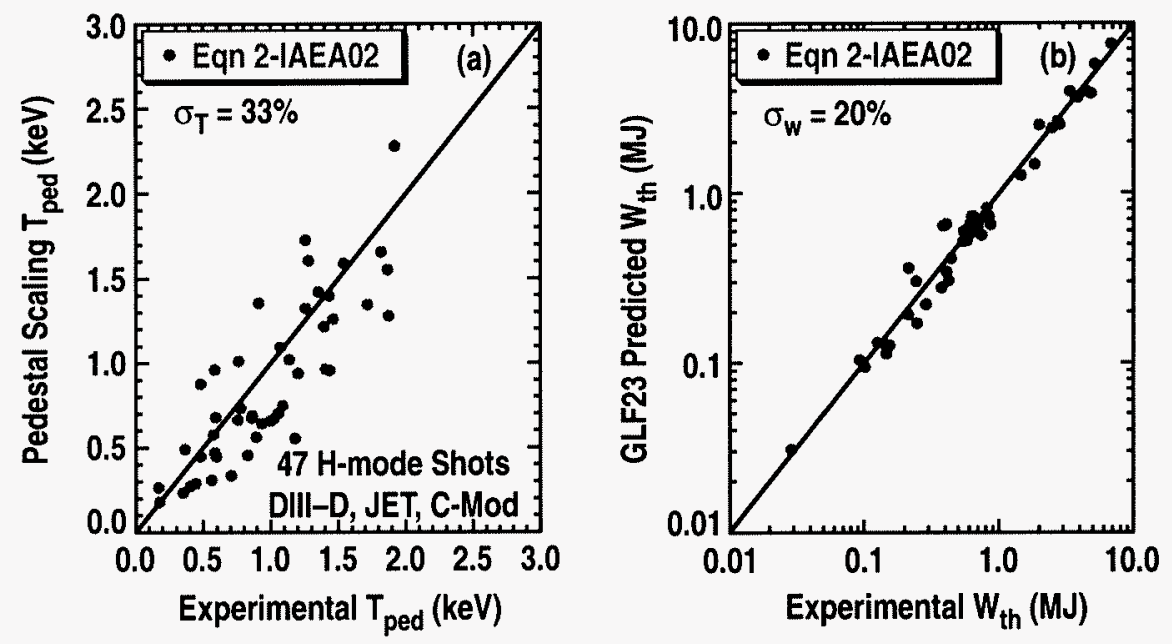

Fig. 3. (a) Predicted versus experimental pedestal temperature using the thermal conduction pedestal scaling from Ref. [6] for $47 \mathrm{H}-$ mode discharges (b) predicted versus experimental core stored energy using the renormed GLF23 model with pedestal scaling boundary conditions.

conditions for $\mathrm{T}_{\mathrm{e}}$ and $\mathrm{T}_{\mathrm{i}}$ in the simulations with GLF23 yields an RMS error $\sigma_{\mathrm{W}}$ of $20 \%$ in the stored energy. The RMS error is defined as $\left[(1 / N) \Sigma\left(W_{S} / W_{x}-1\right)^{2}\right]^{0.5}$ where $W_{S}$ and $W_{x}$ are the simulated and experimental stored energies and $\mathrm{N}$ is the number of discharges. Three DIII-D shots in the 50 shot database were not used since artificial barriers developed near the boundary in the GLF23 simulations when the pedestal scalings were used for $\mathrm{T}_{\text {ped }}$. Here, the boundary conditions were enforced at a normalized radius of $\rho=0.85$. While this may be inside the actual top of the pedestal, $\rho=0.85$ was used to insure that GLF23 was not used in steep gradient pedestal region where it is not applicable. To compensate for this in the pedestal scaling, an enhancement factor $C_{\text {ped }}$ of 1.25 on $T_{\text {ped }}$ was added such that a zero offset in the RMS error in the GLF23 predicted core stored energy using those $T_{\text {ped }}$ values was obtained. For the 47 $H$-mode discharges, including $C_{\text {ped }}$ also changed the average $(1 / N) \Sigma\left(T_{S} / T_{X}\right)$ in the predicted $\mathrm{T}_{\text {ped }}$ from 0.75 to 0.95 .

While using pedestal scalings instead of experimental data for $\mathrm{T}_{\text {ped }}$ made little difference in the RMS error in the predicted temperature profiles using the MM95 model, Fig 3(b) shows that the RMS error in the stored energy for the GLF23 simulations is significantly worse when pedestal scaling boundary conditions are used instead of using the experimental data for $\mathrm{T}_{\text {ped }}$ $\left(\sigma_{\mathrm{W}}=10 \%\right)$. This is likely because GLF23 is a stiffer model compared to MM95 and therefore more sensitive to $\mathrm{T}_{\text {ped. }}$.

The renormalized GLF23 and the original MM95 models have been applied in simulations of the ITER, FIRE, and IGNITOR designs. The design parameters are given in Table 1. The 
predicted fusion performance from simulations using the GLF23 and MM models is shown in Fig. 4 as a function of pedestal temperature assuming a fixed pedestal density, where $\mathrm{n}_{\text {ped }}=0.71 \overline{\mathrm{n}}_{\mathrm{e}}$ and $\overline{\mathrm{n}}_{\mathrm{e}} / \mathrm{n}_{\mathrm{G}}=0.85,0.70$, and 0.60 , respectively for ITER, FIRE, and IGNITOR.

Table 1

Plasma Parameters for ITER, FIRE, and IGNITOR

\begin{tabular}{lccc}
\hline & ITER & FIRE & IGNITOR \\
\hline $\mathbf{R}(\mathbf{m})$ & 6.2 & 2.14 & 1.32 \\
$\mathbf{a}(\mathbf{m})$ & 2.0 & 0.595 & 0.45 \\
$\mathbf{I}_{\mathbf{p}} \mathbf{M A}$ & 15.0 & 7.7 & 9.0 \\
$\mathbf{B}(\mathbf{T})$ & 5.3 & 10 & 13.0 \\
$\mathbf{K}_{95}$ & 1.8 & 1.8 & 1.8 \\
$\boldsymbol{\delta}_{\mathbf{9 5}}$ & 0.4 & 0.4 & 0.4 \\
$\mathbf{n}_{\mathbf{G}}\left(\mathbf{1 0}^{\mathbf{2 0}} \mathbf{m}^{-\mathbf{3})}\right.$ & 1.19 & 6.92 & 14.2 \\
$\overline{\mathbf{n}}_{\mathbf{e}} / \mathbf{n}_{\mathbf{G}}$ & 0.85 & 0.7 & 0.6 \\
$\mathbf{Z}_{\mathbf{e f f}}$ & 1.5 & 1.4 & 1.2 \\
$\mathbf{P}_{\mathbf{a u x}}(\mathbf{M W})$ & 40 & 20 & 10 \\
\hline
\end{tabular}
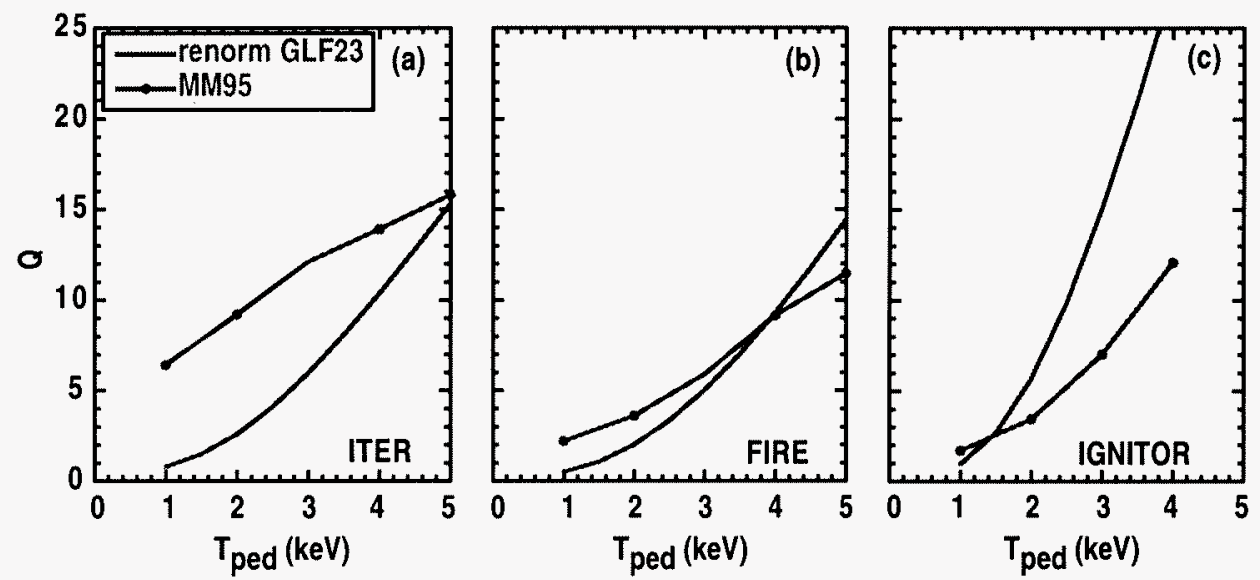

Fig. 4. Fusion Q predicted using MM95 and GLF23 models versus pedestal temperature for (a) ITER (b) FIRE and (c) IGNITOR at fixed pedestal density with $\mathrm{P}_{\mathrm{aux}}=40 \mathrm{MW}$, $20 \mathrm{MW}$, and $10 \mathrm{MW}$ respectively.

The fusion $\mathrm{Q}$ increases with pedestal temperature at fixed plasma density. In general, the renormalized GLF23 model results show a stronger sensitivity to pedestal temperature due to the stiff nature of the model. While the FIRE predictions from the two models are similar, the renormed GLF23 and MM95 models yield very different predictions at low pedestal temperatures for ITER and high pedestal temperatures for IGNITOR. 
Plotting $Q$ versus $T_{\text {ped }}$ at fixed $n_{\text {ped }}$ is misleading, however, since the power independent $\sqrt{ } \beta_{p}$ pedestal model indicates that pedestal temperature is inversely related to pedestal density, as shown in Fig. 5, and the pedestal density is proportional to the core density. Hence, an increase in the plasma density causes a decrease in the pedestal temperature, which partially offsets the gain in fusion power. The pedestal temperatures at the design densities are $2.89,2.82$, and $1.98 \mathrm{keV}$ for ITER, FIRE, and IGNITOR, respectively. Figure 6 shows the resulting fusion Q as a function of normalized plasma density for simulations of ITER, FIRE, and IGNITOR using the renorm GLF23 and MM95 models together with the $R \sqrt{ } \beta_{p}$ and Eq. (2) power dependent pedestal models presented. The results obtained using the power dependent pedestal model (solid lines) leads to more optimistic predictions than those obtained using the MHD limit pedestal model (dashed lines) due to higher values of $\mathrm{T}_{\text {ped }}$. Compared to Fig. 4, the GLF23 and MM95 results for ITER are closer together when the power dependent pedestal scaling is used because the values of $T_{\text {ped }}$ are higher (Fig. 4). At the reference densities, the values for $\beta_{\text {ped }}{ }^{N}\left[\beta_{\text {ped }} /(\mathrm{I} / \mathrm{aB})\right]$ are approximately $0.9,1.3$, and 0.6 for ITER, FIRE, and IGNITOR, respectively, when the GLF23 and MM95 models are used along with the power dependent pedestal scaling (Eq. (2) in Ref. [6]). These results also depend on the assumed auxiliary heating power $\left(\mathrm{Q} \propto \mathrm{P}_{\text {aux }}{ }^{-0.9}\right.$ for renormalized GLF23 and approximately $\mathrm{Q} \propto \mathrm{P}_{\mathrm{aux}}{ }^{-0.5}$ for MM95) and on the impurity concentration.

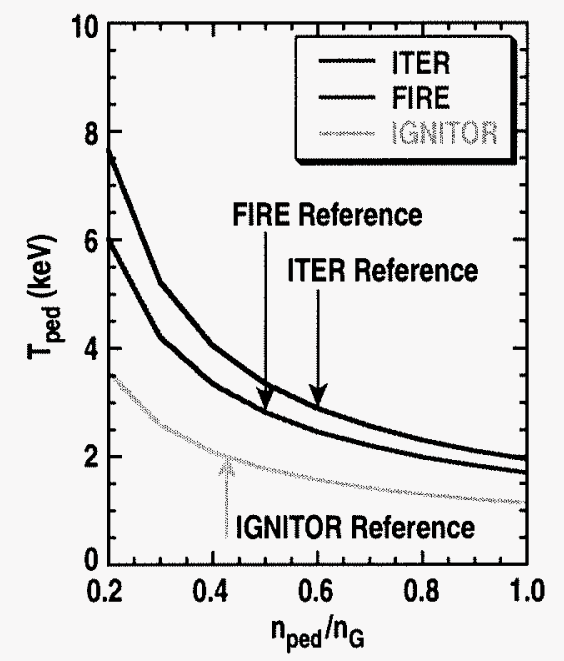

Fig. 5. Predicted pedestal temperature versus pedestal density divided by the Greenwald density $\mathrm{n}_{\mathrm{G}}$ for ITER, FIRE, and IGNITOR using $\mathrm{R} \vee \beta_{\mathrm{p}}$ pedestal model. 

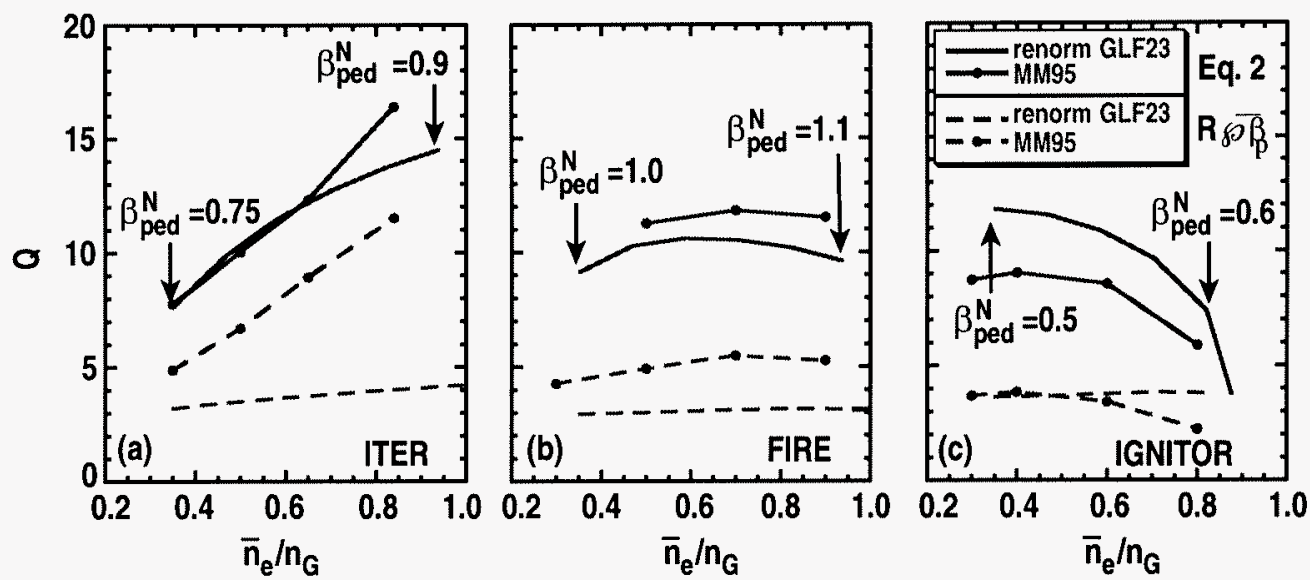

Fig. 6. Fusion Q predicted using MM95 and GLF23 models versus line-averaged electron density divided by the Greenwald density for (a) ITER, (b) FIRE, and (c) IGNITOR assuming Tped varies with the density according to the power dependent (solid lines) and $R \sqrt{ } \beta_{p}$ (dashed lines) pedestal models. 


\section{ACKNOWLEDGMENT}

Work supported by U.S. Department of Energy under Grants DE-FG02-92ER54141 and DEFG03-95ER54309. 


\section{REFERENCES}

[1] R.E. Waltz, G.M. Staebler, W. Dorland, et al., Phys. Plasmas 4, 2482 (1997).

[2] G. Bateman, A.H. Kritz, J.E. Kinsey, et al., Phys. Plasmas 5, 1793 (1998).

[3] R.E. Waltz, J.M. Candy, M.N Rosenbluth, Phys. Plasmas 9, 1938 (2002).

[4] F. Jenko, W. Dorland, et al., Phys. Plasmas 7, 1904 (2000).

[5] T. Onjun, G. Bateman, A.H. Kritz, and G. Hammett, to appear in Phys. Plasmas, December 2002.

[6] ITPA H-mode Database and the Pedestal DatabaseWorking Groups, IAEA-CT-CN94ITERP/02, 19th IAEA Fusion Energy Conference, Lyon, France, 2002; Plasma Phys. Contr. Fus. 44, A429 (2002). 